\title{
Multiple dark matter scenarios from ubiquitous stringy throats
}

\author{
Diego Chialva, ${ }^{1}$ P. S. Bhupal Dev, ${ }^{2}$ and Anupam Mazumdar ${ }^{3,4}$ \\ ${ }^{1}$ Service de Mecanique et Gravitation, Université de Mons, Place du Parc 20, 7000 Mons, Belgium \\ ${ }^{2}$ Consortium for Fundamental Physics, School of Physics and Astronomy, University of Manchester, \\ Manchester M13 9PL, United Kingdom \\ ${ }^{3}$ Consortium for Fundamental Physics, Lancaster University, Lancaster LA1 4YB, United Kingdom \\ ${ }^{4}$ Niels Bohr Institute, Copenhagen University, Blegdamsvej 17, DK-2100, Denmark
}

(Received 8 November 2012; published 22 March 2013)

\begin{abstract}
We discuss the possibility of having multiple Kaluza-Klein dark matter candidates which arise naturally in generic type-IIB string theory compactification scenarios. These dark matter candidates reside in various throats of the Calabi-Yau manifold. In principle, they can come with a varied range of masses in four dimensions depending upon the hierarchical warping of the throats. We show that consistency with cosmological bounds and four-dimensional effective theory description imposes strong constraints on the parameter space and the geometry of the throats. With a rather model-independent approach, we find that the mass scales allowed for the Kaluza-Klein dark matter particles in various throats can vary between $0.1 \mathrm{eV}$ and $10 \mathrm{TeV}$, depending upon the throat geometry. Thus, there could be simultaneously more than one kind of cold (and possibly warm and hot) dark matter components residing in the Universe. This multiple dark matter scenario could weaken the bound on a conventional supersymmetric dark matter candidate and could also account for extra relativistic degrees of freedom in our Universe.
\end{abstract}

DOI: 10.1103/PhysRevD.87.063522

PACS numbers: 95.35.+d, 98.80.Cq, 11.25.Mj

\section{INTRODUCTION}

Several astrophysical and cosmological observations have established the existence of dark matter (DM) in our Universe [1] and, hence, a possible hint for new physics beyond the Standard Model (SM). Although all evidence so far is based only on its gravitational interaction, the combined data from cosmological and particle physics sources require any standard DM candidate to satisfy the following conditions: (i) it must be stable on cosmological time scales and have the right pressure (to help large scale structure formation), (ii) it must have the right relic density (not to overclose the Universe), and (iii) it must be very weakly interacting with the SM particles (to satisfy the direct and indirect detection constraints).

There exist a plethora of particle DM candidates [2] with masses ranging from $10^{-5} \mathrm{eV}$ to as high as the grand unified theory (GUT) scale $\sim 10^{16} \mathrm{GeV}$, motivated by various new physics scenarios. The most studied DM candidates are the weakly interacting massive particles (WIMPs) in the mass range $\sim 1 \mathrm{GeV}-10 \mathrm{TeV}$, as they occur almost naturally in very well-motivated particle physics theories, and have rich phenomenological implications. For example, one of the most popular WIMP candidates is the lightest supersymmetric particle in supersymmetric models with conserved $R$ parity [3] which were originally proposed to provide an elegant solution to the gauge hierarchy problem of the SM.

Various phenomenological extra-dimensional models provide an alternative potential solution to the gauge hierarchy problem, and also a viable WIMP DM candidate in the form of the lightest Kaluza-Klein (KK) mode. In the simplest universal extra-dimensional models, the lightest
KK particle remains stable, and hence a possible DM candidate [4], due to KK parity which is a remnant of the translational invariance in the extra dimension, after orbifolding (i.e., a $Z_{2}$ symmetry) is imposed to obtain chirality. In the more realistic Randall-Sundrum (RS) model [5], where the hierarchy between the electroweak and Planck scales arises naturally from a warped geometry of the extra-dimensional space, the stability of the KK modes can be ensured in a bottom-up approach by imposing a gauged symmetry [6], or in a top-down approach by assuming the (approximate) preservation of isometries [7].

On the other hand, it is desirable to study a model of DM where the DM candidates arise naturally from more complete and fundamental theories capable of also describing, in a coherent and unified framework, the other stages of the evolution of our Universe, such as inflation [8] (for reviews, see, e.g., Refs. $[9,10])$. The superstring theory is arguably the most developed Planck scale theory that can make a phenomenological connection at low scales and, hence, can potentially capture the whole history of evolution of our Universe. It provides a viable description of our four-dimensional spacetime by compactifying six extra dimensions on a Calabi-Yau manifold.

In the so-called flux compactification models, the internal Calabi-Yau manifold presents background fluxes arising from three-forms piercing through the internal cycles, and numerous sources of energy such as orientifold planes, $D$-branes, $\bar{D}$-branes, $N S$-branes, etc. [11]. The fluxes, branes and orientifold planes typically reduce the $\mathcal{N}=2$ supersymmetry preserved by the Calabi-Yau compactification to $\mathcal{N}=1$ or null supersymmetry. The backreaction from the fluxes transforms the metric from a perfectly 
factorized one into a warped geometry. Strongly warped regions develop generically when the fluxes are supported on cycles localized in small regions of the internal manifold. A typical example is the case of cycles that can shrink to a conifold singularity (which can then be deformed), arguably the most generic singularity in Calabi-Yau spaces, which can occur in large numbers due to the presence of many three-cycles.

It is now a prediction of string theory that flux configurations and the generic presence of several conifold points lead to a multiple-throat scenario [12,13]. Such a setup is also phenomenologically preferred as throats with different warpings give rise to different hierarchies, thus solving the hierarchy puzzle in particle physics as well as providing viable inflationary models in the hidden sectors or in the bulk [10,14-16]. However, the string models generically predict many hidden sectors which makes it difficult to obtain the branching ratios among visible and hidden sectors after preheating and reheating.

As a consequence of the multithroat scenario, matter localized in the hidden throats (i.e., different from the throat where the SM resides ${ }^{1}$ ) can represent cosmologically stable DM, as the stability against decaying into SM fields can be ensured by a sufficient suppression of decay rates due to the necessary tunneling between throats in a warped geometry ${ }^{2}[16]$. This, in addition to the usual visible sector DM candidate in the (supersymmetric) SM throat, naturally leads to a multiple DM scenario. ${ }^{3}$ We will consider the case of KK modes in the hidden throats from higher dimensional bulk gravitational fields (which are always present in the models), assuming for simplicity no extra branes and, hence, no other matter content in the hidden throats although any form of matter in these throats could in principle represent a DM candidate. The stability and mass range will depend on the depth of the throats (gravitational redshifting).

The number of string compactifications is very large (dubbed as the "landscape"). Hence, it would be convenient to find a general way to check if the multithroat multiple DM scenario can be effectively realized. In this paper, we show that the relic density of the KK modes in sufficiently long throats has remarkably general features (essentially due to the decoupling of the long throats from each other) and, hence, can be written in a calculable and rather general way without resorting to the fine details of the underlying string model. We also study how other quantities of interest can be analyzed in rather general

\footnotetext{
${ }^{1}$ For simplicity we will not consider the case where the SM resides in the bulk.

${ }^{2}$ Note that this is different from the warped scenarios in Refs. $[7,13]$, where the lightest KK particle resides only within the SM throat and its stability is guaranteed by the (approximate) preservation of isometries of the throat.

${ }^{3}$ For an incomplete list of other multiple DM scenarios in various particle physics models, see, e.g., Refs. [17,18].
}

terms. In this way, we are able to apply all possible bounds coming from (i) cosmology/astrophysics and (ii) consistency of the model description, and derive important constraints on the parameter space and the throat geometry even when the branching ratio of the inflaton to visible and hidden sector degrees of matter cannot be determined so easily from the string top-down approach. We emphasize again that our phenomenological approach is independent of the details of the string inflation models and also of the dynamics of (p)reheating.

We find that there are allowed regions of the parameter space for this multiple DM scenario to work and that some features can be determined rather generically. In particular, we obtain the possible range of masses for the DM candidates residing in various throats, and the bounds on the local string scales. We also discuss the bounds on the inflationary scale for certain classes of models. Finally, we point out some interesting phenomenological consequences of this multiple DM scenario.

The paper is organized as follows: In Sec. II, we discuss the stability of the KK DM candidates in the multithroat scenario. In Sec. III, we calculate their relic density and obtain the bounds on the parameter space for our multiple DM scenario. In Sec. IV, we briefly discuss some interesting phenomenological implications. Our conclusions are given in Sec. V.

\section{STABILITY OF THE KK MODES IN MULTIPLE THROATS}

In the multithroat scenario we are entertaining, the new DM candidates are the KK modes residing in throats different from the SM one. ${ }^{4}$ The stability against decaying to and interacting with the SM particles will be ensured by the smallness of the couplings to the SM fields and, hence, of the decay rates as they are suppressed by the necessity of quantum-mechanical tunneling among the separate throats. Here we summarize the main features of such tunneling, and in the next section we will discuss the constraints on the warping factor of the throats from (i) cosmological constraints, (ii) consistency of the description and (iii) the requirements of stability and correct cosmic abundance of these KK modes.

The local metric for each throat far from the tip can be written as a warped product with the generic form

$d s^{2}=H(r)^{-1 / 2}\left(g_{\mu \nu} d x^{\mu} d x^{\nu}\right)+H(r)^{1 / 2}\left(d r^{2}+r^{2} d s_{X_{5}}^{2}\right)$,

where $\mu, \nu=0,1,2,3$ run through the four-dimensional metric. The radial coordinate $r$ reaches a minimum value of $r_{\min }$ at the tip of the throat, and the local string scale at the tip of the $i$ th throat is given by

$$
\left.M_{i} \equiv M_{s} H\left(r_{\min }\right)^{-1 / 4}\right|_{i \text { th throat }} \equiv M_{s} h_{i},
$$

\footnotetext{
${ }^{4}$ We will comment on the possibility of having long-lived KK modes also in the SM throat in Sec. III.
} 
where $h_{i}$ is the maximum warping factor of the $i$ th throat ( $h_{i}=1$ corresponds to no warping), and $M_{s}=\ell_{s}^{-1}$ is the 10-dimensional string mass scale which is usually taken to be smaller than the reduced Planck mass $M_{\mathrm{PL}}=\ell_{\mathrm{PL}}^{-1}=$ $2.4 \times 10^{18} \mathrm{GeV}$.

The lightest KK mass will be given by [13]

$$
m_{\mathrm{KK}(i)} \sim \frac{h_{i}}{R_{i}},
$$

where $R_{i} \geq \ell_{s}$ is the curvature radius of the $i$ th throat. The spacing between the KK masses is also given by $\frac{h_{i}}{R_{i}}$. From the four-dimensional point of view, the largest mass which we can imagine will be always bounded by the compactification scale, while the lowest KK mass can be very small (see, e.g., Refs. [19]) and is limited only by the consistency of the model.

Quantum-mechanical tunneling will occur among the separate throats as long as the quantum numbers of the tunneling modes match in the two throats. The decay rate depends on the equation of motion of the modes, and thus on the specific form of the metric (1) generating the gravitational potential wall between the throats.

Different modelings of the compactified geometry with different forms of the warping factor $H(r)$ in Eq. (1) or modelings of the bulk region between throats lead to different tunneling rates. The prototypical throat metric is the Klebanov-Strassler solution [20] for which, however, the exact solution of the mode equation is not known. Useful approximations, which are adapted to different scenarios, have been used in the literature in order to obtain the decay rate. When the throat can be approximated as an $\operatorname{AdS}_{5} \times$ $X_{5}$, and the bulk region connecting two throats as a "Planck brane" akin to the case of the RS model [5], the tunneling rate from the $i$ th throat to any other neighboring throat can be estimated to be [13]

$$
\Gamma_{\mathrm{tunn}(i)}^{(\mathrm{RS})}=\left(m_{\mathrm{KK}(i)} R_{i}\right)^{4} \frac{r_{\min _{(i)}}}{R_{i}^{2}} \sim \frac{h_{i}^{5}}{R_{i}} .
$$

Other modelings of the compactified geometry worked out in the literature lead in general to more suppressed decay rates. For example those in Ref. [16] present two possibilities giving rise to the decay rates

$$
\Gamma_{\text {tunn(i) }}^{(\mathrm{I})} \sim h_{i}^{9} R_{i}^{-1} \quad \text { and } \quad \Gamma_{\text {tunn }(i)}^{(\mathrm{II})} \sim h_{i}^{17} R_{i}^{-1} .
$$

For concreteness, we will present our results considering these models as exemplary possible realizations in the landscape of compactifications, and investigating what constraints on the string models follow from the cosmological and consistency requirements for a suitable multithroat DM scenario.

To be a DM candidate the KK modes in the hidden throats must be stable. Typically models require preserved isometries to guarantee perfectly stable DM candidates. However, isometries in string throat compactifications are generically broken because of the gluing of the throats to the bulk [21]. Despite this, we learn from Eq. (4) that tunneling from a longer throat (small warping $h_{i}$ ) to a shorter one is highly disfavored, and therefore the interactions of the KK modes in such throat with matter localized in other throats (as the SM or KK modes in other throats) will be suppressed (small overlapping of wave functions). For what concerns the DM properties, this means that the lowest KK mode in the $i$ th throat will be stable against interacting with matter localized in other throats provided that the throat is long enough to ensure that the tunneling lifetime is larger than the age of the Universe, i.e.,

$$
\Gamma_{\text {tunn }(i)} \lesssim H_{0},
$$

where $H_{0}=100 h \mathrm{~km} \cdot \mathrm{s}^{-1} \cdot \mathrm{Mpc}^{-1}$, and the parameter $h$ has been measured by WMAP to be $h=0.72 \pm 0.03$ [22].

The KK modes in any given throat could still be unstable due to (i) self-interactions, i.e., interactions with matter localized in the same throat, and (ii) annihilation into massless bulk modes (gravitons). ${ }^{5}$ For what concerns the interactions (i), as already noted in the introduction, for a simple viable multiple DM scenario, we have assumed the hidden throats not to harbor any matter content other than the KK modes which self-interact, with a suppression scale given essentially by the local string scale $[15,16]$ because of their localization, until they freeze out and eventually become DM relics. In Sec. III, we will see that, in the absence of any isometries making them absolutely stable, this process is very rapid for the values of parameters compatible with suitable DM scenarios and that essentially only lowest KK modes remain in the hidden throats as DM relics. In the case of the visible throat (containing the SM degrees of freedom), the localized KK modes should rapidly decay into the SM radiation to provide a standard cosmology with radiation domination at early times. This will also be discussed in Sec. III.

On the other hand, the annihilation into massless bulk modes (ii) is suppressed because of two reasons [15,16]: First, the annihilation cross section of the KK modes to gravitons is Planck-suppressed as $\sigma_{g} \sim M_{\mathrm{PL}}^{-2}$ (whereas the KK self-interactions are warp-enhanced, and thus essentially suppressed by the local string scale $\sigma_{K K} \sim g_{s}^{2} M_{i}^{-2}$ ), and further reduced by the fact that only modes carrying oppositely conserved internal momentum can annihilate. Second, gravitons are rapidly redshifting ( $\sim a^{-4}, a$ being the scale factor) compared to the massive KK modes $\left(\sim a^{-3}\right)$, when the latter become nonrelativistic. Therefore, the gravitational interactions

\footnotetext{
${ }^{5}$ The decay $1 K K_{(i)} \rightarrow 2$ gravitons is forbidden by KaluzaKlein number conservation for our gravitational $\mathrm{KK}$ nonzero modes. The annihilation $2 K K_{(i)} \rightarrow 1$ graviton gravitonturns out then to be the dominant interaction left to consider, as we have already discussed the tunneling effects that suppress the interactions involving modes from different throats or the SM.
} 
become ineffective soon. This also ensures that gravitons are not overproduced [16].

Thus we conclude that in the multithroat scenario considered here, ensuring the condition (6) is sufficient for the stability of the KK modes in a given throat. Ensuring that condition will put constraints on the geometry of the compactifications.

\section{BOUNDS AND RELIC DENSITY OF THE KK DARK MATTER}

The production of the KK modes localized in various throats depends on the stringy realization of inflation and (p)reheating. However, the postproduction evolution of the KK modes in the hidden throats shows remarkable model-independent features, because of the particular background geometry leading to the tunneling suppression of the interaction among different throats.

This scenario has a striking difference compared to the standard WIMP DM picture. In the latter case, the DM candidate participates in the same thermal history as the SM degrees of freedom and evolves independently only after freeze-out (which determines its relic density). Instead, in the multithroat scenario, the DM particles in different long enough throats are decoupled from each other and from the SM degrees of freedom by tunneling suppression of the interaction cross sections between them. ${ }^{6}$ As shown later in this section, their relic density is determined essentially by the initial conditions. ${ }^{7}$

Such dependence on the initial conditions of production can be efficiently parametrized by subdividing the models into two general classes, as discussed below, on the basis of the mechanism of reheating and production of the KK modes. Therefore, our analysis can be rather modelindependent and adapt for the landscape setting.

In one class of models, the energy which excites the KK modes in the hidden throats and reheats the SM in its own throat is initially localized essentially in a single (non-SM) throat, from which it is transferred to the others by tunneling. A typical example is the $D-\bar{D}$ inflation models with the mechanism of reheating-throughtunneling out of the inflationary throat studied in Refs. $[15,16]$. Note however that our analysis is independent of the specific models, as appropriate for the landscape: it applies to all models where the energy responsible for reheating of the DM and SM fields

\footnotetext{
${ }^{6}$ Gravitational interactions, though not tunneling suppressed, are suppressed by $M_{\mathrm{PL}}^{2}$ and hence unable to lead to thermal equilibrium among the throats.

${ }^{7}$ In the multithroat scenario, the DM in each throat has its own thermal history, and in case it reaches thermal equilibrium in the throat, its own temperature. These possible temperature differences might affect the counting of the total relativistic degrees of freedom $g_{*}$ and $g_{* S}$ entering, respectively, the formulas for the energy density and entropy. However, the dependence of the relic density on these quantities will turn out to be weak.
}

resides initially in a single throat, independently of the details of how and where inflation occurred. We will call this throat the "initial throat" (indicated with the label "in"), and this class of models "throat reheating." 8

In the other class of models, which we call "bulk reheating," the energy responsible for reheating does not reside in one specific initial throat, but is spread over the internal manifold. Typical examples are those where the reheating field is a bulk inflaton or a bulk modulus, which decays releasing its energy all over the warped Calabi-Yau manifold. These models, however, must reheat the SM efficiently, therefore requiring different branching ratios among the throats, and also among the bulk and the throats. Note again that our analysis will be independent of specific models in this case too. It may even be the case that inflation has occurred in one specific throat, but couples more efficiently to bulk moduli or fields such as gravitinos, which then, through their decay, will reheat the other degrees of freedom from the bulk.

As our analysis will be independent of the specific features of perturbative reheating or preheating, we use the term "reheating" in a generic way, encompassing all possible ways that produce the KK modes within the hidden throats.

Summarizing, the essential features of the models relevant for our analysis are (1) sufficiently long throats are decoupled from each other because of tunneling suppression, and (2) the models can be divided in two broad classes depending on if (2A) the energy responsible for reheating is initially localized in one specific throat from where it has to tunnel out, or if (2B) the energy responsible for reheating can be released through direct couplings and does not require tunneling to excite the other degrees of freedom.

\section{A. Reheating in one throat}

In this scenario the energy density responsible for reheating is initially localized in one specific throat from where it has to tunnel out. The typical example is the scenario of $D-\bar{D}$ inflation in one throat. In that case, the end of inflation is brought about by the annihilation of the $D$ - and $\bar{D}$-branes. Their decay products are massive fundamental closed strings, which decay until KK modes that quickly thermalize are left over $[15,16]$. Then these modes tunnel to the other throats reheating them. ${ }^{9}$

\footnotetext{
${ }^{8} \mathrm{We}$ will not consider the case when the SM and the initial throat coincide, as the analysis in this case is similar to that in Refs. [6,7] for the RS model.

${ }^{9}$ The modes at tunneling are nonrelativistic. Indeed, if we assumed that they were relativistic, the corresponding temperature $T_{\text {in,tunn }}$ would be found to be smaller than the mass of even the lightest KK mode $\left(m_{K K(\text { in })}\right)$. For example, even for the less suppressed decay rate in the case of RS-like throat geometry, the ratio is $\frac{T_{\text {in,tunn }}}{m_{K K(\text { in })}}=\sqrt{R_{\mathrm{in}} M_{\mathrm{PL}}} h_{\mathrm{in}}^{3 / 2}$, which is generally much smaller than one.
} 
The largest part of the energy will be tunneled into the longest throat since it has the densest spectrum, which favors tunneling as the level matching is easier and more energy levels fall within the width of the initial tunneling mode. The remaining degrees of freedom end up in sufficiently long throats since the tunneling out of them is very suppressed. The shallow throats, having a less dense spectrum, less likely receive the energy tunneling from the initial throat, and moreover the received energy will anyway tunnel out of them in a short time scale due to their large tunneling rates. Hence, the shallow throats can be practically treated as empty for the purposes of our analysis.

Note that since the initial throat has to be shallower than the other throats for tunneling to occur, even the lightest modes in the initial throat will tunnel to modes which are not the lightest ones in their respective throats because of the level matching. These higher modes in the final (ith) throat are in general unstable and decay into the lighter ones much more rapidly than the Hubble rate after tunneling from the initial (in) throat provided that the self-interaction rate of the KK modes in the $i$ th throat $\Gamma_{\mathrm{SM}}=\frac{m_{i}^{3}}{M_{i}^{2}}$ is larger than the Hubble rate right after tunneling, ${ }^{10}$ i.e.,

$$
h_{\mathrm{in}}^{3}>R_{\mathrm{in}}^{2} M_{i}^{2} \sqrt{R_{\mathrm{in}}^{2} M_{\mathrm{PL}} \Gamma_{\mathrm{in}, \mathrm{tunn}}},
$$

which, as we will see later, is easily satisfied for the parameter space leading to allowed DM models. Hence, we can safely assume that the long hidden throats harbor only DM candidates in the form of the lightest KK modes.

Assuming that the SM radiation dominates soon after tunneling and is in thermal equilibrium (the necessary conditions will be discussed later), as envisaged in a standard cosmology with radiation domination at early times followed by matter domination, and recalling that the different long enough throats are decoupled from each other by tunneling suppression, we can derive an equation for the current relic density of the KK dark matter candidates in the hidden throats:

$$
\begin{aligned}
\Omega_{X} h^{2} & =\frac{1.3 \times 10^{-4}}{g_{*}^{\prime} g_{*}\left(T_{\mathrm{in}, \mathrm{tunn}}\right)} \frac{\sum_{i} m_{X_{i}} n_{X_{i}}\left(t_{\mathrm{in}, \text { tunn }}\right)}{T_{\mathrm{in}, \mathrm{tunn}}^{3} T_{0}} \\
& =\frac{1.8 \times 10^{8}}{g_{*}^{\prime}}\left(\frac{T_{\mathrm{in}, \mathrm{tunn}}}{1 \mathrm{GeV}}\right) F_{X} .
\end{aligned}
$$

Here, $T_{0}$ is today's temperature, $t_{\mathrm{in}, \text { tunn }} / T_{\mathrm{in} \text {,tunn }}$ is the time/ temperature after tunneling from the initial throat, $g_{*}^{\prime} \equiv$ $\frac{g_{*}\left(T_{0}\right) g_{* S}\left(T_{\text {int,unn }}\right)}{g_{* S}\left(T_{0}\right) g_{*}\left(T_{\text {in, tunn }}\right)}$ (with $g_{*}, g_{* S}$ the total relativistic degrees of freedom entering, respectively, the relation for energy density and entropy), $n_{i}(t)$ is the number density of the DM particles $X_{i}$, and

\footnotetext{
${ }^{10}$ Due to level matching, the mass of the tunneled KK modes is $m_{i} \sim m_{\mathrm{KK}(\mathrm{in})}$.
}

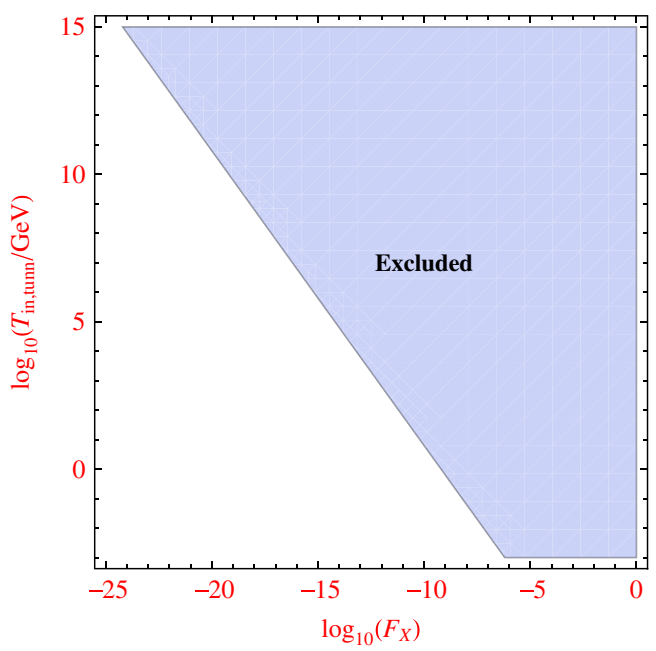

FIG. 1 (color online). The allowed parameter space satisfying the overclosure constraint. We have taken $g_{* S}\left(T_{\text {in,tunn }}\right) \simeq$ $g_{*}\left(T_{\text {in,tunn }}\right)=250$.

$$
F_{X} \equiv \sum_{i}\left(\frac{m_{X_{i}}}{m_{K K(\mathrm{in})}}\right)\left(\frac{n_{X_{i}}\left(t_{\mathrm{in}, \mathrm{tunn}}\right)}{n_{\mathrm{tot}}\left(T_{\mathrm{in}, \mathrm{tunn}}\right)}\right)
$$

is the sum of the branching ratios after tunneling from the initial throat for the $X_{i}$ modes in the throats $i \neq\{\mathrm{SM}$, in $\}$ weighted by the ratio of their masses $m_{X_{i}}$ and the lightest KK mass $m_{K K(\text { in) }}$ in the initial throat. ${ }^{11}$ Note that the sum in Eq. (9) includes only the non-SM long throats; the dynamics of Kaluza-Klein modes in the SM throat requires a different analysis that takes into account the interactions within the SM fields, as shown later in this section.

In order to avoid overclosure of the Universe by the KK dark matter particles, we require the relic density given by Eq. (8) to be less than the $3 \sigma$ upper limit of the WMAP observed value [22]: $\Omega_{X} h^{2} \leq 0.13$. The allowed range of $F_{X}$ satisfying this condition is shown in Fig. 1. This result will be used later in this section to constrain the parameter space for a multiple DM scenario. As the dependence on the total numbers of relativistic degrees of freedom at different temperatures is not strong, we have assumed the common value 250 at early times, to account for the minimal supersymmetric SM sector plus some hidden degrees of freedom.

The condition of SM radiation domination after tunneling from the initial throat requires the SM throat to be the one which receives more energy from the initial tunneling. A necessary condition for this is that the SM throat must have the densest spectrum, which favors tunneling as the matching between energy levels is easier (tunneling is

\footnotetext{
${ }^{11}$ Note that in deriving Eq. (8) we do not make any assumption (relativistic/nonrelativistic or even thermal/nonthermal) on the energy or number density of the modes $X_{i}$ at $t_{\text {in,tunn }}$. The final expression of the relic density in Eq. (8) is however similar to those in Refs. [23,24].
} 
favored when the final energy eigenstates fall within the width of the initial state). This implies

$$
h_{i} R_{i}^{-1}>h_{\mathrm{SM}} R_{\mathrm{SM}}^{-1}
$$

The radiation domination further depends on the thermal history of the degrees of freedom in the SM throat. The modes tunneled there can (1) decay into the lowest KK (SM) modes, (2) thermalize, (3) decay into SM fields. In the allowed range of warping, the decay into the SM radiation $[15,25]$ is very rapid, i.e., less than one Hubble time $\sim H_{\text {in,tunn }}^{-1} \sim \Gamma_{\text {in,tunn }}^{-1}$ right after they have tunneled, provided that

$$
\Gamma_{\mathrm{SM}}=\frac{m_{\mathrm{KK}, \mathrm{SM}}^{3}}{M_{\mathrm{SM}}^{2}}=h_{\mathrm{SM}} \frac{\ell_{S}^{2}}{R_{\mathrm{SM}}^{3}} \gg \Gamma_{\mathrm{in}, \mathrm{tunn}} .
$$

Because of Eq. (10), this also entails a lower bound on the warping of the hidden throats. Note that the breaking of isometries due to the gluing of the throat to the bulk [21] ensures the decay of the Kaluza-Klein modes in the Standard Model throat.

It is possible that mildly broken isometries could exist at the tip of the initial throat [26]. In this case, the KK(in) modes possessing the related approximately conserved quantum number would be long-lived (LL) modes. They would not easily tunnel out because of difficult quantum number matching between throats, so that the other DM and SM throats will not be populated by these dangerous LL modes. Their present relic density reads

$$
\Omega_{\mathrm{LL}_{\text {in }}} h^{2}=\frac{3.24 \times 10^{26}}{g_{s}^{2} \tilde{g}_{*} \bar{g}_{*}} \frac{M_{\mathrm{in}}^{2}}{M_{\mathrm{PL}}^{2}} \frac{T_{\mathrm{in}, \mathrm{tunn}}}{m_{X_{\mathrm{in}}}}\left(\frac{m_{\mathrm{LL}_{\text {in }}}}{T_{\mathrm{dec}, \mathrm{LL}_{\mathrm{in}}}}\right)^{\frac{3}{2}},
$$

where $T_{\mathrm{dec}, \mathrm{LL}_{\mathrm{in}}}$ is the decoupling temperature of the LL modes with mass $m_{\mathrm{LL}_{\text {in }}}$, given by

$$
\frac{m_{\mathrm{LL}_{\text {in }}}}{T_{\mathrm{dec}, \mathrm{LL}_{\mathrm{in}}}}=\log \left(\frac{\sqrt{3}}{(2 \pi)^{3 / 2}} g_{*}\left(T_{\mathrm{dec}, \mathrm{LL}_{\mathrm{in}}}\right)^{\frac{1}{2}} g_{s}^{2} \frac{M_{\mathrm{PL}} m_{\mathrm{LL}_{\mathrm{in}}}}{M_{\mathrm{in}}^{2}}\right),
$$

and $M_{\text {in }}$ is the local string scale in the initial throat. We have also defined

$$
\begin{gathered}
\tilde{g}_{*} \equiv \frac{g_{* S}\left(T_{\mathrm{dec}, \mathrm{LL}_{\mathrm{in}}}\right) g_{*}\left(T_{\mathrm{RDMD}}\right)^{\frac{3}{4}}}{g_{*}\left(T_{\mathrm{dec}, \mathrm{LL}}\right)^{\frac{1}{2}} g_{* S}\left(T_{\mathrm{RDMD}}\right)}, \\
\bar{g}_{*} \equiv \frac{g_{*}\left(T_{\mathrm{in}, \mathrm{MD}}\right) g_{* S}\left(T_{\mathrm{in}, \mathrm{tunn}}\right)}{g_{* S}\left(T_{\mathrm{in}, \mathrm{MD}}\right) g_{*}\left(T_{\mathrm{in}, \mathrm{tunn}}\right)},
\end{gathered}
$$

where $T_{\mathrm{in}, \mathrm{MD}}$ is the temperature when the lightest $\mathrm{KK}$ mode in the initial throat becomes nonrelativistic, and $T_{\mathrm{RDMD}}$ is the temperature at the standard transition between radiation and matter domination. Equations (12) and (13) match the standard result for cold relics [27], except for the additional suppression factor $\left(\bar{g}_{*}\right)^{-1} \frac{T_{\text {in,tunn }}}{m_{X_{\text {in }}}}$ due to the matter domination (MD) period between the time when the lightest $\mathrm{KK}$ modes in the initial throat become nonrelativistic and the end of tunneling from the initial throat.

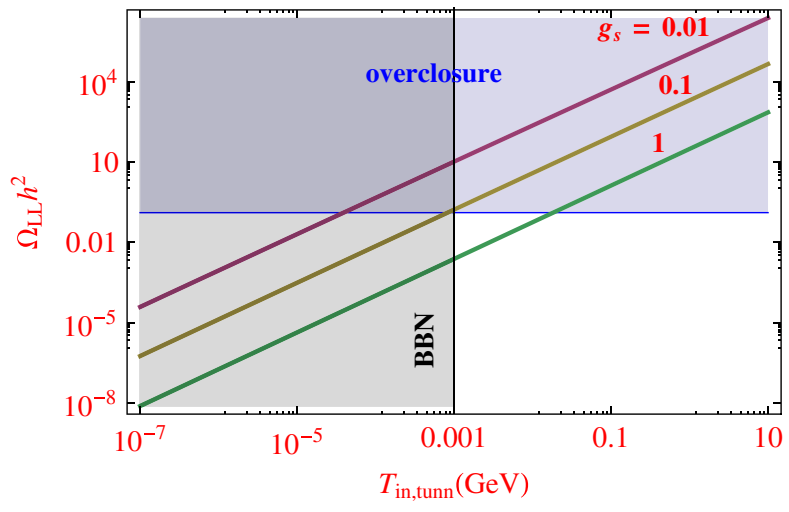

FIG. 2 (color online). The relic density of the long-lived KK modes in the initial throat with RS-like geometry as a function of the tunneling temperature. Here we have chosen $R_{\text {in }}=10 \ell_{s}$ and $M_{s}=10^{-2} M_{\mathrm{PL}}$. The vertical shaded region is excluded from BBN constraints. In the horizontal shaded region, these LL modes will overclose the Universe. Note that for the string coupling $g_{s} \leq 0.1$, these LL modes, if present, are always dangerous relics.

To illustrate our point on the LL modes, the relic density (12) for the LL(in) modes is plotted in Fig. 2 for some typical string coupling values. In this plot, we show the case where the throat geometry is well approximated by the $\mathrm{AdS}_{5} \times X_{5}$ RS-like picture, and we have used $R_{\text {in }}=10 \ell_{s}$ and $M_{s} \sim 10^{16} \mathrm{GeV}$ (GUT scale). As before, due to the weak dependence on the total numbers of relativistic degrees of freedom at different temperatures, we have assumed the common value 250 at early times.

We see that unless the interactions of these LL modes are strong enough (for $g_{s} \gtrsim 1$ ), their relic density will be too large in the allowed temperature range, i.e., $T_{\mathrm{in}, \mathrm{tunn}}$, $T_{\text {dec, } \mathrm{LL}_{\text {in }}}$ larger than the big bang nucleosynthesis (BBN) temperature $\sim 1 \mathrm{MeV}$ [27]. Thus, we conclude that for reasonably small values of the string coupling $g_{s} \leq 0.1$ we must not have the long-lived KK modes in the initial throat. In other words, all the isometries must be broken (which is anyway expected by the junction of the throats to the bulk [21]) in order for the Universe not to be overclosed. Increasing the value of $R_{\text {in }}$ worsens the situation, and only a lower value of $M_{s}$ would improve it. For the cases where the decay rate is more suppressed, as in the models of Ref. [16], the constraint for the LL modes is even more difficult to satisfy; for instance, for the values of the parameters chosen for Fig. 2, even the case $g_{s}=1$ is ruled out for the LL modes.

Besides overclosure, there are other cosmological constraints to be satisfied as well. First of all, the tunneling decay must occur well before the BBN in the SM throat which happens at temperature $T_{\mathrm{BBN}} \sim 1 \mathrm{MeV}$, and sets the lower bound for $T_{\mathrm{in}, \mathrm{tunn}}$. We should mention here that this is the only robust lower bound we can put on the tunneling (and hence, reheating) temperature since we do not yet know for sure the exact history of the observable Universe 

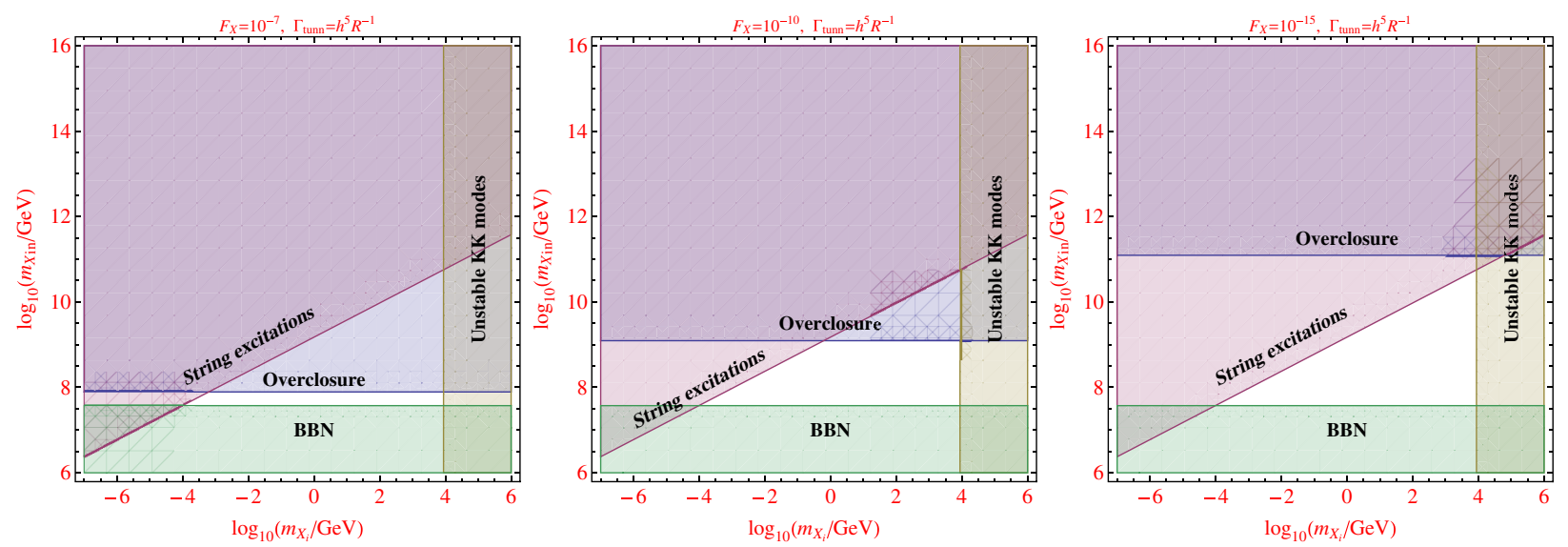

FIG. 3 (color online). Examples of allowed ranges of parameters, shown as the white (unshaded) region, for the class of models discussed in Sec. III A for different fractions $F_{X}$ defined by Eq. (9) and arbitrary number of throats in terms of the lightest KK masses in the initial throat $\left(m_{X_{\text {in }}}\right)$ and other hidden throats $\left(m_{X_{i}}\right)$. We plot here the case of $\mathrm{AdS}_{5} \times X_{5}$ RS-like throat geometry (for other cases see text). Here, we have chosen the string scale to be $M_{\mathrm{PL}} / 100$ and the AdS radius of curvature in each throat to be $10 M_{\mathrm{PL}}^{-1}$, where $M_{\mathrm{PL}}=2.4 \times 10^{18} \mathrm{GeV}$ is the reduced Planck mass. Note that the condition (10) in combination with (11) is automatically satisfied for these examples and we have not shown it in the plot.

before the epoch of BBN. In practice, such extreme values for reheating temperature could be problematic for successful baryogenesis (see however Ref. [28] for an example where this could still work). In such cases, the allowed parameter space to be shown in the following figures might shrink considerably.

Furthermore, for our four-dimensional field theory description to be valid, we should have the temperature $T_{\text {in,tunn }}$ lower than the local string scale in each throat in order to avoid exciting stringy degrees of freedom, which entails a lower bound on the local string scale (or, on the warp factor, and thus, on the KK mass) in each throat:

$$
M_{i} \gtrsim T_{\mathrm{in}, \text { tunn }} \Rightarrow m_{X_{i}} \geq \frac{\ell_{s}}{R_{i}} T_{\mathrm{in,tunn}} .
$$

It may be noted here that the more realistic lower bound on the warp factor might be obtained by considering the scale of throat deformation/decompactification due to moduli destabilization. However, this is very hard to compute explicitly in the Hubble expansion background [29,30], and hence, we use the more intuitive (and in general weaker) bound in Eq. (16) using the string scale.

Summarizing, the bound from BBN temperature, the condition Eq. (16), the relic density overclosure constraint, the requirements of rapid and efficient SM reheating [i.e., Eqs. (10) and (11)], and the stability condition (6) in each throat are the conditions that need to be satisfied in any working multithroat DM model. These in turn put strong constraints on the warping factors of the throats harboring the KK modes, which are directly related to the DM masses $m_{X_{i}}$ and the local string scales $M_{i}$ in the throats.

We have studied the allowed region of parameter space after imposing these conditions. The stringy parameters in the landscape setting are warpings, throat curvatures, scales, tunnelings rates, and initial branching ratios.
Through the above equations and discussion, we have related them in a rather model-independent way (that is, independently of the fine details of the compactification) to the constrained quantities such as relic densities and KK masses. The relation between the warping $h_{\text {in }}$ and the tunneling temperature $T_{\text {in,tunn }}$ follows from the dependence of $\Gamma_{\text {in,tunn }}$ on $h_{\text {in }}$, which in turns depends on the specific form of the throat metric. As mentioned before, we will consider the decay rates in Eqs. (4) and (5) as exemplary model realizations.

For illustration, we present in Fig. 3 the results, for different fractions $F_{X}$ and certain values of the string and throat curvature scales, for the case when the throat geometry is well approximated by a RS-like $\mathrm{AdS}_{5} \times X_{5}$ picture, and will discuss later the results for other parameter values and models with more suppressed tunnelings as those in Ref. [16].

In Fig. 3 the constraints are shown as various shaded regions and the allowed parameter space after imposing all the constraints is shown as the white (unshaded) region. We see that the size of the allowed region (which gives a measure of the fine-tuning) depends on the value of $F_{X}$ in the landscape of compactifications, but some features of the working models (such as the DM mass range) do not. We have used $R_{i} \simeq R_{\text {in }} \simeq R=10 \ell_{s}$ and $M_{s} \sim 10^{16} \mathrm{GeV}$ (GUT scale). ${ }^{12}$ With the above choices, the allowed mass range for the DM candidates is limited to about

\footnotetext{
${ }^{12}$ The assumption that the curvature radius $R$ is similar for all throats is justified by the fact that in string theory flux compactification typically $R \sim(F)^{\frac{1}{4}} g_{s}^{\frac{4}{4}} \ell_{s}[11,20,31]$ (this is certainly true when the throat metric is of the $\mathrm{AdS}_{5} \times X_{5}$ RS-like kind), where $F$ is given by some flux/brane numbers which can vary from throat to throat. Even if $F$ varies by a factor $10^{6}, R$ differs just by about one order of magnitude.
} 
$0.1 \mathrm{MeV}-10 \mathrm{TeV}$, with the local string scale in the initial throat at most at $\sim 10^{12} \mathrm{GeV}$. Note that the condition (10) in combination with (11) is automatically satisfied for the cases shown in Fig. 3 and we have not shown it in the plot. However, this constraint is generally important in the other cases, discussed below.

In the case of models where the tunneling rate is more suppressed by higher powers of the warping than in the case of RS-like geometry of the throats (see Sec. II), the allowed region of parameter space shrinks, and the warping of the initial throat has to be shallower; i.e., $m_{X_{\text {in }}}$ and $M_{\text {in }}$ have to be larger. For the most suppressed case with $\Gamma \sim$ $h^{17} R^{-1}$ for the throats, the mass scales are found to be quite restricted; for instance, $m_{X_{\text {in }}}$ can only vary within about an order of magnitude.

As mentioned, we have also studied how the allowed region of parameter space varies with the parameters $R$ and $M_{s}$. Keeping the latter fixed and letting $R$ vary, the allowed region shrinks (both for RS-like throat geometry and for the cases with more suppressed decay rates) and moves to lower mass scales. However, we find that the mass of the DM candidates cannot be lower than about $0.1 \mathrm{eV}$ satisfying all the constraints along with the condition in Eq. (11). Also, keeping $R$ fixed, the upper bounds on the KK mass scales in the initial and other hidden throats get lower when $M_{s}$ gets smaller (without affecting much the lower bound on $m_{X_{i}}$ ), and the allowed region again shrinks.

Interestingly, when the throat geometry is well approximated by an $\mathrm{AdS}_{5} \times X_{5}$ RS-like picture, for all values of $R$ and $M_{s}$, we find that no allowed region exists for the local string scale in the initial throat $M_{\text {in }}$ exceeding about $10^{13} \mathrm{GeV}$ in the compactified effective theory (i.e., with stabilized throat geometry). If inflation takes place in this throat, then its scale cannot be larger than the local string one, and this would rule out a high scale (around GUT scale) multithroat inflationary scenario in this case. This in turn puts a strong constraint on the tensor-to-scalar ratio and, hence, the possibility of detecting primordial gravitational waves (see, e.g., Ref. [9]).

\section{B. Reheating in the bulk}

In this scenario the energy density responsible for reheating is not localized in a throat and couples without tunneling suppression to all throats. Although this initial energy density can be stored in bulk modes or moduli fields different from the inflaton, for simplicity we will call "inflatons" all the degrees of freedom associated with the initial energy density. The reheating process in this kind of model can lead to various effects, including destabilization of the geometry. For our purposes, however, we can start our analysis from when the throats have again settled down and the perturbative Kaluza-Klein picture is valid. We assume that the KK modes have been produced by the inflaton reheating process at a reheat temperature $T_{R}$ within the throats where they are localized.
As discussed earlier, the $\mathrm{KK}$ modes in different long throats will be decoupled from each other because of tunneling suppression of the relevant interaction cross sections. The present DM abundance can be estimated, assuming SM radiation domination after reheating, and without making assumptions regarding the energy/number density of the $X_{i}$ modes at the reheating time $t_{R}$. We obtain a result similar to Eq. (8), with $T_{\text {in,tunn }}$ replaced by $T_{R}$ and $F_{X}=\frac{\sum_{i} m_{X_{i}} n_{X_{i}}\left(t_{\mathrm{R}}\right)}{\rho_{\text {tot }}\left(T_{\mathrm{R}}\right)}$, where $\rho_{\text {tot }}\left(T_{\mathrm{R}}\right)=0.3 g_{*}\left(T_{R}\right) T_{R}^{4}$.

Differently from the throat-reheating scenario, in the bulk-reheating case we are discussing here, the SM fields can be reheated directly since the "inflaton" is directly coupled to them. In this case, we do not have to require the condition (11) for SM radiation to be produced rapidly. However, the inflaton is coupled to the other throats as well and with the bulk; hence, there could easily be overproduction of the non-SM degrees of freedom and/or gravitons. The efficiency of these processes is however modeldependent and beyond the scope of our discussion here. ${ }^{13}$

Another important difference with the throat-reheating case concerns the possibility of long-lived modes because of mildly broken isometries [26]. We recall that in the throat-reheating case all the energy density comes from the initial throat by tunneling, and LL modes in that throat will not easily tunnel out because of difficult quantum number matching. Thus, the DM and SM throat will not be populated by dangerous long-lived KK modes. But in the case of bulk reheating, dangerous LL modes can be excited in all throats, if mildly broken isometries exist, due to the direct coupling with the reheating (inflaton) sector. Their relic density in the SM throat has the same form as Eq. (12) without the suppression factor due to matter domination before tunneling, and substituting all "in" labels with the label "SM" indicating the throat in question:

$$
\Omega_{\mathrm{LL}_{\mathrm{SM}}} h^{2}=\frac{3.24 \times 10^{26}}{g_{s}^{2} \tilde{g}_{*}} \frac{M_{\mathrm{SM}}^{2}}{M_{\mathrm{PL}}^{2}}\left(\frac{m_{\mathrm{LL}_{\mathrm{SM}}}}{T_{\mathrm{dec}, \mathrm{LL}}}\right)^{\frac{3}{2}},
$$

where the decoupling temperature is obtained by similarly substituting the "in" labels with "SM" in Eq. (13). From Eq. (16), we can see that in the presence of LL modes in the SM throat, the overclosure bound will constrain the warping factor $h_{\mathrm{SM}}$. For instance, for $M_{s} \sim 10^{16} \mathrm{GeV}, g_{s} \sim 0.1$ and $R_{\mathrm{SM}}=10 \ell_{s}$, we get $h_{\mathrm{SM}} \lesssim 10^{-14}$. The bound gets stronger if the interactions of the LL modes are weaker (i.e., $g_{s}$ is smaller or $M_{s}$ is increased). For each of the other hidden throats, the $\operatorname{LL}(\mathrm{j})$ relic density will be determined by the effective decoupling among throats due to tunneling suppression.

\footnotetext{
${ }^{13}$ For instance, in large volume compactification models, it is well-known that the inflaton branching ratio to the hidden degrees is much more than the visible sector degrees of freedom [32].
} 

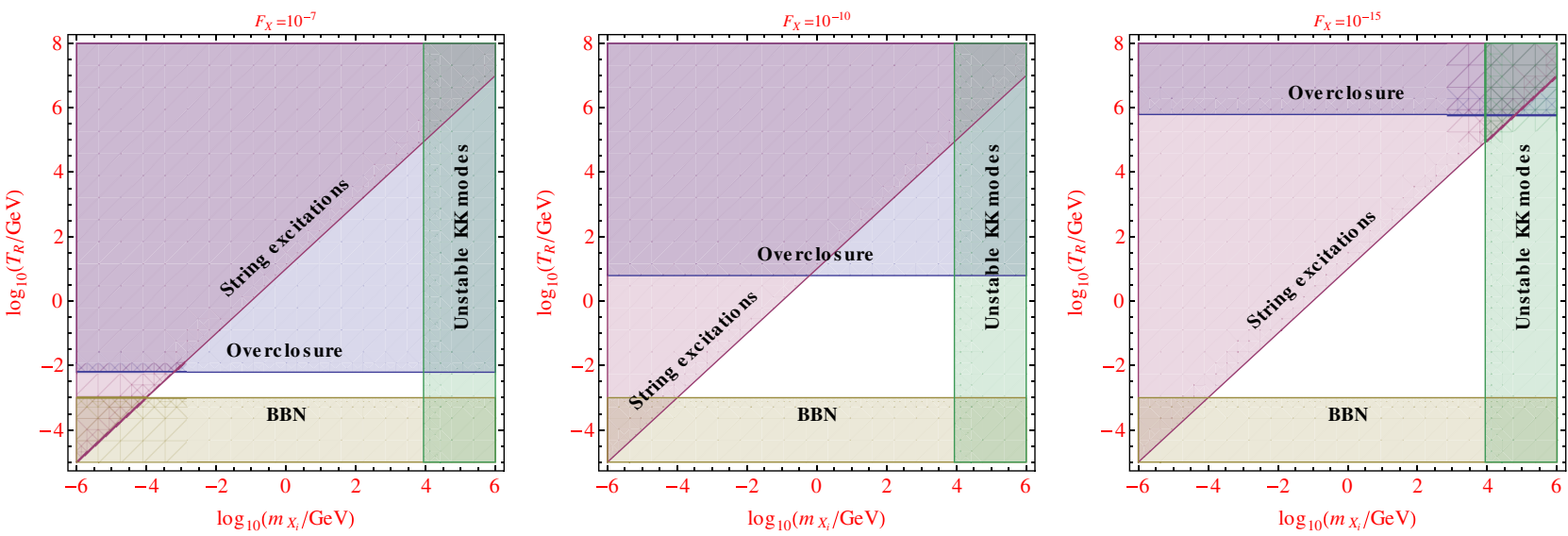

FIG. 4 (color online). Examples of the allowed range, shown as the white (unshaded) region, of the reheating temperature $\left(T_{R}\right)$ and the lightest KK mass in the hidden throats $\left(m_{X_{i}}\right)$ in the bulk-reheating case (Sec. III B) for different energy density fractions $F_{X}$ and arbitrary number of throats. We plot here the case of $\mathrm{AdS}_{5} \times X_{5}$ RS-like throat geometry (for other cases see text). Here we have chosen the string scale to be $M_{\mathrm{PL}} / 100$ and the radius of curvature in each throat to be $10 M_{\mathrm{PL}}^{-1}$, where $M_{\mathrm{PL}}=2.4 \times 10^{18} \mathrm{GeV}$ is the reduced Planck mass.

We have therefore two scenarios for the requirement that stable KK modes do not overclose the Universe in the bulkreheating case, depending on if the mildly broken isometries responsible for the presence of long-lived modes exist or not. In the latter case, the total relic density is just $\Omega_{\mathrm{X}} h^{2}$ given by Eq. (8) with $t_{\text {in,tunn }} / T_{\text {in,tunn }}$ replaced by $t_{R} / T_{R}$. But if there are some isometries and the relative LL modes, the total relic density is obtained by including in $F_{\mathrm{X}}$ the number density and mass of the $\operatorname{LL}(\mathrm{j})$ modes.

As before, we ask here for the temperature $T_{R}$ to be lower than the string scale in the throats, to avoid exciting stringy degrees of freedom, which entails $M_{i} \geqslant T_{R}$. This, together with the relic density overclosure constraint and the requirement of stability of the KK modes, leads to bounds on the mass scale of the KK modes.

We first discuss the results for the case when the throat geometry is well approximated by an $\mathrm{AdS}_{5} \times X_{5}$ RS-like picture. For illustration, this case is shown in Fig. 4 for the choice $R_{i} \simeq R_{\text {in }}=10 \ell_{s}$ and $M_{s} \sim 10^{16} \mathrm{GeV}$. We further note that in the bulk-reheating case, if there are long-lived $\mathrm{KK}$ modes in the throats, the allowed mass range for the $\mathrm{KK}$ modes is even smaller, i.e., between $0.1 \mathrm{MeV}$ and $100 \mathrm{GeV}$.

Varying $R_{i}$ and $M_{s}$, or considering the non-RS-like geometries such as in Ref. [16], leads to the same variation of scales and shrinking of the allowed region discussed in the previous subsection. However in this case, it is not possible to obtain in a model-independent manner a minimum absolute lower bound on $m_{X_{i}}$ or a relation between the maximum $T_{R}$ and the scale of inflation.

\section{Number of DM candidates and fine-tuning}

Finally, we present an estimate of the total number of the KK dark matter candidates for the multithroat scenarios we have discussed. From the top-down approach, given a number $C$ of conifold points in the Calabi-Yau manifold, the average number of throats with a warping $h<\bar{h}$ is [12]

$$
\bar{N}(h<\bar{h} \mid C) \simeq \frac{C}{3 \log (1 / \bar{h})} .
$$

Using the upper bound $h_{\max }$ on the warping factor for a given throat to harbor the stable KK modes as derived with the above analysis, we obtain $\bar{N}\left(h<h_{\max } \mid C\right) \sim 0.01 C$; i.e., the number of dark matter candidates in our multithroat scenario is roughly $1 \%$ of the total number of conifold points in the Calabi-Yau manifold which depends on the underlying string compactification model. This also gives a measure of the fine-tuning necessary to obtain the suitable models of DM we have been considering.

\section{SOME PHENOMENOLOGICAL IMPLICATIONS}

In this section, we briefly discuss some interesting phenomenological consequences of the multiple DM scenario advocated here. Our discussion in this section will be qualitative only, since we cannot present precise quantitative predictions without resorting to a particular model. For detailed quantitative aspects of the multiple DM phenomenology, see, e.g., Refs. [17,18,33].

(i) The total DM relic density will be given by the sum of the relic densities in the visible and hidden sectors:

$$
\Omega_{\mathrm{DM}}^{\text {total }} h^{2}=\Omega_{\mathrm{DM}}^{\text {visible }} h^{2}+\Omega_{\mathrm{DM}}^{\text {hidden }} h^{2},
$$

where $\Omega_{\mathrm{DM}}^{\text {hidden }} h^{2}$ includes the contribution of the KK dark matter particles and, possibly, other stable and weakly interacting matter from other hidden sectors. This implies that the lower limit of the WMAP measured value, $\Omega_{\mathrm{CDM}} h^{2}>0.09$ [22], is no more applicable to the visible sector DM candidate. 
This releases a large part of the parameter space in many visible sector DM models. For example, this provides one way to salvage the constrained minimal supersymmetric SM with the neutralino lightest supersymmetric particle in the light of the recent LHC data, even though the additional parameter space available for $\Omega h^{2}<0.09$ [34] is not spectacularly large.

(ii) The KK dark matter candidates in our multithroat scenario are allowed to be light and, hence, could possibly serve as warm (or even hot) dark matter candidates (if their velocity distribution is the opportune one). If the KK modes are sufficiently light (of order $\mathrm{eV}$ ), they could act as extra sterile degrees of freedom (similarly to sterile neutrinos [35]) from the point of view of the visible sector, thus possibly explaining the deviation of the measured value of the effective number of relativistic degrees of freedom at $\mathrm{BBN}, N_{\mathrm{eff}}^{\mathrm{WMAP}}=4.34 \pm 0.87$ [22], from the $\mathrm{SM}$ value $N_{\mathrm{eff}}^{\mathrm{SM}}=3$. One of the main constraints on the number of sterile degrees of freedom is provided by the high sensitivity of the expansion rate of the Universe at the BBN temperature: $T_{\mathrm{SM}, \mathrm{BBN}} \sim$ $1 \mathrm{MeV}$. Sufficiently light KK modes could change the expansion rate of the Universe and thereby impact the BBN, even though they do not reside in the SM throat, and do not have any SM interactions. This can be translated into a bound (see, e.g., Ref. [2]),

$$
g_{\mathrm{KK}}\left(\frac{T_{\mathrm{KK}, \mathrm{BBN}}}{T_{\mathrm{SM}, \mathrm{BBN}}}\right)^{4}=\frac{7}{8} \times 2 \times\left(N_{\mathrm{eff}}-3\right),
$$

where $g_{\mathrm{KK}}$ is the number of internal degrees of freedom for the relativistic KK modes. This bound could be satisfied in our scenario depending on the precise value of the KK dark matter temperature in the hidden throats.

(iii) In the scenario where inflation occurs in the initial throat, the bounds on the local mass scale also constrain the scale of inflation in the compactified effective theory (for which we must have $R_{i}>\ell_{S}$, $M_{s}<M_{\mathrm{PL}}$ ). In particular, when the throat geometry is well approximated by the $\mathrm{AdS}_{5} \times X_{5}$ RS-like picture, the local string scale, and hence the scale of inflation, in the initial throat is found to be $\lesssim 10^{13} \mathrm{GeV}$. This would put a strong constraint on building any inflationary model with large tensor-toscalar ratio, which would generate detectable gravitational waves during or after inflation (see, e.g., Ref. [9]). Therefore, if such a large tensor-to-scalar ratio is observed (e.g., by the Planck satellite), this would certainly disfavor the multithroat warped models with RS-like throat geometry where inflation occurs in a hidden throat, since the Universe will be inevitably overclosed by the KK relics.

(iv) Depending on the model construction, the KK modes residing in the SM throat could decay to light supergravity modes and/or directly to the SM particles, thus leading to some interesting indirect detection signals $[7,36]$. Very light modes in the SM throat could also provide observable effects for example in the gravity sector, and hence, a test for our model.

\section{CONCLUSION}

We have discussed the possibility of having multiple dark matter candidates in the form of the lightest KK modes residing in various long throats which arise naturally out of generic Calabi-Yau compactification in string theories. The stability of these KK modes in long enough throats is due to the small tunneling rate because of the background warped geometry. Compliance with cosmological and consistency constraints imposes strong bounds on the underlying string compactified models. However, we have shown that there exists some parameter space where the KK modes can be DM candidates. We have been able to study these features in a rather model-independent way, and thus in the optic of a string landscape analysis.

This gives us a very different picture for particle dark matter than the usually discussed single WIMP scenario. We also note here that relaxing the absolute cosmological stability constraint for the DM states and instead requiring only a dynamical balance against their abundance, as in the so-called dynamical DM scenario [18], might open up a larger parameter space in our case.

As a word of caution, we must keep in mind the generality of the models we have considered, by neglecting the precise couplings between the reheating and the reheated degrees of freedoms in the bulk-reheating scenario, which preclude us from knowing the exact branching ratios, and assuming pure KK matter content in the hidden throats and not other possible, more complicated matter sectors as well as radiation. We leave some of these issues for future investigation.

\section{ACKNOWLEDGMENTS}

We thank the referee for a critical reading of the manuscript and for many useful comments. The work of D.C. is supported by the Belgian National "Fond de la Recherche Scientifique" F.R.S.-F.N.R.S. with a contract "chargé de recherche." The work of B.D. and A.M. is supported by the Lancaster-Manchester-Sheffield Consortium for Fundamental Physics under STFC Grant No. ST/J000418/1. 
[1] See, e.g., G. Bertone, D. Hooper, and J. Silk, Phys. Rep. 405, 279 (2005).

[2] See, e.g., J. L. Feng, Annu. Rev. Astron. Astrophys. 48, 495 (2010).

[3] See, e.g., G. Jungman, M. Kamionkowski, and K. Griest, Phys. Rep. 267, 195 (1996).

[4] G. Servant and T.M. P. Tait, Nucl. Phys. B650, 391 (2003); H.-C. Cheng, J.L. Feng, and K. T. Matchev, Phys. Rev. Lett. 89, 211301 (2002).

[5] L. Randall and R. Sundrum, Phys. Rev. Lett. 83, 3370 (1999); 83, 4690 (1999).

[6] K. Agashe and G. Servant, Phys. Rev. Lett. 93, 231805 (2004).

[7] A. R. Frey, R. J. Danos, and J. M. Cline, J. High Energy Phys. 11 (2009) 102.

[8] R. Allahverdi, B. Dutta, and A. Mazumdar, Phys. Rev. D 75, 075018 (2007); Phys. Rev. Lett. 99, 261301 (2007); C. Boehm, J. Da Silva, A. Mazumdar, and E. Pukartas, Phys. Rev. D 87, 023529 (2013).

[9] A. Mazumdar and J. Rocher, Phys. Rep. 497, 85 (2011).

[10] L. McAllister and E. Silverstein, Gen. Relativ. Gravit. 40, 565 (2008).

[11] See, e.g., M. Grana, Phys. Rep. 423, 91 (2006); M. R. Douglas and S. Kachru, Rev. Mod. Phys. 79, 733 (2007).

[12] A. Hebecker and J. March-Russell, Nucl. Phys. B781, 99 (2007).

[13] S. Dimopoulos, S. Kachru, N. Kaloper, A. E. Lawrence, and E. Silverstein, Int. J. Mod. Phys. A 19, 2657 (2004).

[14] N. Barnaby, C. P. Burgess, and J. M. Cline, J. Cosmol. Astropart. Phys. 04 (2005) 007.

[15] D. Chialva, G. Shiu, and B. Underwood, J. High Energy Phys. 01 (2006) 014.

[16] X. Chen and S.-H. H. Tye, J. Cosmol. Astropart. Phys. 06 (2006) 011.

[17] C. Boehm, P. Fayet, and J. Silk, Phys. Rev. D 69, 101302 (2004); A. Aguirre and M. Tegmark, J. Cosmol. Astropart. Phys. 01 (2005) 003; T. Hur, H.-S. Lee, and S. Nasri, Phys. Rev. D 77, 015008 (2008); Q.-H. Cao, E. Ma, J. Wudka, and C.-P. Yuan, arXiv:0711.3881; B. von Harling and A. Hebecker, J. High Energy Phys. 05 (2008) 031; J. L. Feng and J. Kumar, Phys. Rev. Lett. 101, 231301 (2008); K. M. Zurek, Phys. Rev. D 79, 115002 (2009); D. E. Morrissey, D. Poland, and K. M. Zurek, J. High Energy Phys. 07 (2009) 050; I. Cholis and N. Weiner, arXiv:0911.4954; D. Feldman, Z. Liu, P. Nath, and G. Peim, Phys. Rev. D 81, 095017 (2010); P. T. Winslow, K. Sigurdson, and J. N. Ng, Phys. Rev. D 82, 023512 (2010); B. Batell, Phys. Rev. D 83, 035006 (2011); Z.-P. Liu, Y.-L. Wu, and Y.-F. Zhou, Eur. Phys. J. C 71, 1749 (2011); G. Belanger, K. Kannike,
A. Pukhov, and M. Raidal, J. Cosmol. Astropart. Phys. 04 (2012) 010; M. Aoki, M. Duerr, J. Kubo, and H. Takano, Phys. Rev. D 86, 076015 (2012).

[18] K. R. Dienes and B. Thomas, Phys. Rev. D 85, 083523 (2012); 85, 083524 (2012); 86, 055013 (2012); K. R. Dienes, S. Su, and B. Thomas, Phys. Rev. D 86, 054008 (2012); K. R. Dienes, J. Kumar, and B. Thomas, Phys. Rev. D 86, 055016 (2012).

[19] S. Panda, Y. Sumitomo, and S. P. Trivedi, Phys. Rev. D 83, 083506 (2011).

[20] I. R. Klebanov and M. J. Strassler, J. High Energy Phys. 08 (2000) 052.

[21] A. Berndsen, J. M. Cline, and H. Stoica, Phys. Rev. D 77, 123522 (2008).

[22] E. Komatsu et al. (WMAP Collaboration), Astrophys. J. Suppl. Ser. 192, 18 (2011).

[23] A. H. Campos, L. L. Lengruber, H. C. Reis, R. Rosenfeld, and R. Sato, Mod. Phys. Lett. A 17, 2179 (2002).

[24] R. Allahverdi and M. Drees, Phys. Rev. Lett. 89, 091302 (2002); Phys. Rev. D 66, 063513 (2002).

[25] S. Chang and M. Yamaguchi, arXiv:hep-ph/9909523; T. Han, J. D. Lykken, and R.-J. Zhang, Phys. Rev. D 59, 105006 (1999); H. Davoudiasl, J. L. Hewett, and T. G. Rizzo, Phys. Rev. Lett. 84, 2080 (2000); K. Cheung, Phys. Rev. D 63, 056007 (2001).

[26] L. Kofman and P. Yi, Phys. Rev. D 72, 106001 (2005).

[27] E.W. Kolb and M.S. Turner, The Early Universe, Frontiers in Physics (Westview Press, Boulder, 1989).

[28] S. Davidson, M. Losada, and A. Riotto, Phys. Rev. Lett. 84, 4284 (2000).

[29] S. B. Giddings and A. Maharana, Phys. Rev. D 73, 126003 (2006).

[30] A. R. Frey, A. Mazumdar, and R. C. Myers, Phys. Rev. D 73, 026003 (2006).

[31] S. B. Giddings, S. Kachru, and J. Polchinski, Phys. Rev. D 66, 106006 (2002).

[32] M. Cicoli and A. Mazumdar, J. Cosmol. Astropart. Phys. 09 (2010) 025; Phys. Rev. D 83, 063527 (2011).

[33] S. Profumo, K. Sigurdson, and L. Ubaldi, J. Cosmol. Astropart. Phys. 12 (2009) 016; A. V. Macciò, O. Ruchayskiy, A. Boyarsky, and J.C. Munoz-Cuartas, Mon. Not. R. Astron. Soc. 428, 882 (2013); M. Baldi, Ann. Phys. (Berlin) 524, 602 (2012); arXiv:1206.2348.

[34] H. Baer, V. Barger, and A. Mustafayev, J. High Energy Phys. 05 (2012) 091.

[35] B. Gripaios, Nucl. Phys. B768, 157 (2007); B830, 390(E) (2010); K. Kadota, Phys. Rev. D 77, 063509 (2008).

[36] X. Chen, J. Cosmol. Astropart. Phys. 09 (2009) 029. 